\title{
Managing anticoagulation in the COVID-19 era between lockdown and reopening phases: Comment
}

\author{
Arianna Magon $^{1}$ (1) $\cdot$ Cristina Arrigoni ${ }^{2} \cdot$ Serena Barello ${ }^{3} \cdot$ Guendalina Graffigna $^{3} \cdot$ Rosario Caruso $^{4}$
}

Received: 22 December 2020 / Accepted: 15 January 2021 / Published online: 10 February 2021

(c) Società Italiana di Medicina Interna (SIMI) 2021

Keywords Anticoagulation $\cdot$ COVID-19 $\cdot$ Medication adherence $\cdot$ Public health $\cdot$ Patient engagement

Dear editor

We read with great interest a recent contribution published by Poli et al. (2020), highlighting the impacts of the COVID-19 pandemic on therapeutic choices in managing oral anticoagulation therapy (OAC). We would like to add some comments and reflections on the specific impact that the restriction measures adopted for flattening the epidemic curve of the COVID-19 pandemic have on strategies for managing outpatients in OAC. We strongly agree with the authors' suggestions of favoring anticoagulation with direct oral anticoagulants (DOACs) in patients without contraindications, and to promote the use of point-of-care (POC) devices in those on vitamin K antagonists (VKAs) [1]. To add evidence to these suggestions and support their implementation, we report some data from the interim analysis we have conducted on a National Italian study aimed to describe independent risk factors of non-adherence among patients on OAC during the COVID-19 pandemic. So far, we enrolled 258 patients during the epidemic tail-end of the first Italian epidemic wave (June-August 2020). At the beginning of the lockdown due to the COVID-19 outbreak in Italy (March-May 2020), the Italian Federation of Anticoagulation Clinics (FCSA) published statements to offer advice for preventing person-to-person diffusion [1]. Based on

Arianna Magon

arianna.magon@grupposandonato.it

1 Department of Biomedicine and Prevention, University of Rome Tor Vergata, Via Agadir, 20-24 - 20097 San Donato Milanese (Mi), Rome, Italy

2 Department of Public Health, Experimental and Forensic Medicine, Section of Hygiene, University of Pavia, Pavia, Italy

3 Department of Psychology, EngageMinds HUB, Consumer and Health Research Center, Università Cattolica del Sacro Cuore, Milan, Italy

4 Health Professions Research and Development Unit, IRCCS Policlinico San Donato, San Donato Milanese, Milano, Italy the statements of the FCSA, Poli et al. (2020) provided practical guidance for improving patient care during both lockdown and reopening phases (June-August 2020). In particular, some actions have been identified for the reopening phase (tail-end of the first Italian epidemic wave), such as maintening all recommended precaution for ensuring safety for staff and patients, rapid and active clinical and laboratory follow-ups, and switch from AVK to DOAC when feasible [1].

In our interim analysis, among the subgroup of patients that could be theoretically switched from VKAs to DOACs $(n=207)$, only 23 were switched. Patients under VKAs were 222 , and only 81 practised a self-management model of OAC. Among the patients who reported to adopt a self-management model, the majority (74.3\%) did not receive either any educational training in self-management or any proposal to attend educational initiatives. However, our survey suggests that patients are generally willing to be involved in educational initiatives for best managing OAC: $72.4 \%$ of patients treated with VKAs and traditional OAC model (105 out of 145 patients) are willing to self-manage their treatment during the restriction measures derived from the COVID-19 pandemic. Anyhow, a relevant barrier to self-management is the high cost of POC, which is defined as "unsustainable" from $43.5 \%$ of patients. Finally, considering patients under DOACs $(n=36), 33 \%$ of them $(n=12)$ did not receive adequate information on the management and clinical features of DOACs.

These results reflect a system-related difficulty in receiving the recommendations for best practice in managing OAC [1], more than reflecting the clinician-level decision-making in adhering to those recommendations. In other words, from a public health perspective, there is an emerging mismatch between the pivotal strategies to manage OAC during this pandemic situation (e.g., safe therapeutic switch and implementation of self-management models when a switch from VKAs to DOACs is not feasible) and the availability of public healthcare services. The main public healthcare services 
should support the identified strategies to face the impact of COVID-19 on OAC, for example, encompassing the reimbursements for POC devices and supporting educational initiatives in the continuum from primary care to tertiary care.

We recognize that the COVID-19 pandemic is triggering the need to establish valid and reliable determinants for identifying patients with a higher likelihood of being adherent to OAC when switched from VKAs to DOACs. Although some research has been conducted to define non-modifiable determinants of treatment adherence in patients switched from VKAs to DOACs (e.g., age and previous complications), very little has been done to determine the strength of modifiable determinants, such as health literacy and health engagement, in predicting the adherence to OAC [2, 3]. Precisely, health literacy was described as the individual's capacity to obtain and understand health information to engage in proper decision-making for using health services. Health engagement was described as the process of an emotional and motivational reframing of an individual's role in perceiving the need of managing a health risk condition and its consequences, evolving from being a passive user of services to an active partner of the healthcare system and healthcare professionals [4]. Health engagement has been proven to be effective in explaining chronic patients ability to cope with self-management in the COVID-19 pandemic [5]. In our interim analysis, we tested the associations between health literacy and health engagement with the dichotomic outcome labelled as dysfunctional behaviours used as a proxy assessment of treatment adherence: the interaction of health engagement on the association from health literacy to dysfunctional behaviours was significant, decreasing the odds of dysfunctional behaviours by $26 \%$. This emerging evidence implies that health engagement and health literacy should play strategic roles in the choices regarding thrombosis-hemostasis [3, 4]. For this reason, adequate public healthcare services to deliver evidence-grounded educational initiatives are as essential as the availability of clinical recommendations for optimal OAC management during the COVID-19 pandemic.

In conclusion, we would like to point out that there is a bold need to aligning more than ever the clinical recommendations for optimal OAC management during the COVID-19 pandemic with the available public healthcare services in the different national contexts. This alignment is essential for those national contexts, where public health services struggle to consider educational programs for self-management as an integral part of their clinical care delivery. Our results and previous studies $[3,4]$ support the hypothesis that educational initiatives for sustaining adherence and engagement to OAC should encompass psycho-social strategies for leading patients from being passive users of services or therapies to clinicians' active and engaged partners. In other words, a higher integration between public health sciences, social and psychological disciplines, and clinical perspective is the necessary action and system plan to achieve realist positive impacts in managing oral anticoagulation in chronic patients during the COVID19 pandemic. Nonetheless, we also speculate that switcing patients from VKAs to DOACs or adopting self-management models for managing $\mathrm{OAC}$ without adequate infrastructural support could paradoxically jeopardize patient safety and anticoagulation control.

Acknowledgements We wish to extend our thanks to all participants, patient associations, and scientific societies that supported the initiative, such as the Italian Federation of Anticoagulation Clinics (FCSA), Italian federation of anticoagulated patients associations (Feder-AIPA), Anticoagulazione.it, and Italian association of adult congenital heart disease (AICCA). A special acknowledgement to Dr. Federica Dellafiore and Dr. Tiziana Nania for their contribution in conducting the survey used to support with data our comment.

Funding This research was partially supported by "Ricerca Corrente" funding from the Italian Ministry of Health to IRCCS Policlinico San Donato.

\section{Compliance with ethical standards}

Conflicts of interest The authors declare that they have not conflict of interest.

Statements on human and animal rights The study was approved by the Internal Review Board of IRCCS Policlinico San Donato.

Informed consent All participants provided informed consent prior to their participation.

\section{References}

1. Poli D, Tosetto A, Palareti G et al (2020) Managing anticoagulation in the COVID-19 era between lockdown and reopening phases. Intern Emerg Med 15:783-786. https://doi.org/10.1007/s11739-02002391-3

2. Toorop MMA, Rein N, Nierman MC et al (2020) Self-reported therapy adherence and predictors for nonadherence in patients who switched from vitamin $\mathrm{K}$ antagonists to direct oral anticoagulants. Res Pract Thromb Haemost 4:586-593. https://doi.org/10.1002/ rth2.12316

3. Magon A, Arrigoni C, Moia M et al (2020) Determinants of healthrelated quality of life: A cross-sectional investigation in physicianmanaged anticoagulated patients using Vitamin $\mathrm{K}$ antagonists. Health Qual Life Outcomes 18:1-9. https://doi.org/10.1186/s1295 5-020-01326-y

4. Barello S, Palamenghi L, Graffigna G (2020) The mediating role of the patient health engagement model on the relationship between patient perceived autonomy supportive healthcare climate and health literacy skills. Int J Environ Res Public Health. https://doi. org/10.3390/ijerph17051741

5. Castellini G, Savarese M, Leone S et al (2020) Italian ibd patients coping with covid-19 emergency: The mitigating role of psychological readiness to engage in self-care. Inflamm Bowel Dis 26:E130 E131. https://doi.org/10.1093/ibd/izaa185

Publisher's Note Springer Nature remains neutral with regard to jurisdictional claims in published maps and institutional affiliations. 